\title{
Impacto en la educación primaria tras la emergencia sanitaria ocasionada por la pandemia del COVID-19
}

Enviado: 10 de diciembre de 2020 / Aceptado: 29 de diciembre de 2020

Publicado: 30 de diciembre de 2020

KATHERINE BELÉN CHACHA MURILLO

\section{RESUMEN}

El objetivo central de esta investigación fue analizar el impacto en la educación primaria tras la metodología virtual por la emergencia sanitaria ocasionada por la pandemia COVID-19. El estudio parte de la problemática detectada en el ámbito educativo tras la transición de una educación presencial a una enseñanza virtual. El enfoque de la investigación es mixto, se aplicó una encuesta a los padres de familia de la escuela fiscal Kiwanis perteneciente a un sector rural de la parroquia de Machachi, los resultados se tabularon y se realizó un análisis e interpretación de los datos. Según los datos obtenidos, existen cambios significativos en la educación primaria del sector rural, siendo un reto para los padres de familia, ya que ellos tienen un bajo nivel de educación y la mayoría no cuentan con herramientas tecnológicas y acceso al Internet.

Palabras Clave: educación virtual, medios tecnológicos, pandemia, proceso de enseñanza-aprendizaje, sector rural.

\section{ABSTRACT}

Impact on primary education after the health emergency caused by the COVID-19 pandemic

The health emergency caused by the COVID-19 pandemic has had a significant impact on education systems worldwide as it faces several challenges. The research is based on the problems detected in the educational field after the transition from face-to-face education to virtual teaching. The research approach is mixed, a survey was applied to the parents of the Kiwanis fiscal school belonging to a rural sector of the parish of Machachi, the results were tabulated and an analysis and interpretation of the data was carried out. The main objective of this research is to analyze the impact on primary education after the health emergency caused by the COVID-19 pandemic. According to the data obtained, there are significant changes in primary education in the rural sector, being a challenge for parents, since they have a low level of education and most do not have technological tools and internet access.

Keywords: virtual education, technological media, pandemic, teaching process-learning, rural sector. 


\section{INTRODUCCIÓN}

En la actualidad la emergencia sanitaria ocasionada por la pandemia de COVID-19, notificada por primera vez en Wuhan (China) el 31 de diciembre del 2019 (Organización Mundial de la Salud OMS, 2019) ha traído cambios en el ámbito social, económico y sobre todo educativo, tras la recomendación de las autoridades del sector salud "quédate en casa", como estrategia de prevención y salvaguardar la vida de las personas e intentar disminuir la probabilidad de contagios, lo que ha implicado que el sector educativo despliegue acciones conducentes para continuar con el ciclo escolar vigente.

Los coronavirus $(\mathrm{CoV})$ son una gran familia de virus que causan enfermedades que van desde el resfriado común hasta enfermedades más graves. La epidemia de COVID-19 fue declarada por la OMS una emergencia de salud pública de internacional el 30 de enero de 2020. El Director General de la Organización Mundial de la Salud (OMS), el doctor Tedros Adhanom Ghebreyesus, anunció el 11 de marzo de 2020 que la nueva enfermedad por el coronavirus 2019 (COVID-19) puede caracterizarse como una pandemia. La caracterización de pandemia significa que la epidemia se ha extendido por varios países, continentes o todo el mundo, y que afecta a un gran número de personas (Organización Panamericana de Salud OPS, 2020).

Ante el cese de actividades académicas anunciadas el 12 de marzo del 2020 por la Ministra de Educación Monserrat Creamer (Ministerio de Educación MINEDUC, 2020) la comunidad educativa se mantuvo en jaque, hasta que el 16 de marzo la ministra presentó el Plan Educativo Covid-19, el cual contiene los lineamientos para docentes, directivos, administrativos, Departamentos de Consejo Estudiantil DECE y las Unidades Distritales de Apoyo a la Inclusión UDAI en la educación fiscal de la Sierra y Amazonía (Ministerio de Educación MINEDUC, 2020), mientras que por su parte la Secretaría de Educación del Distrito Metropolitano de Quito también se hacía presente con los lineamientos para la implementación del Plan Educativo Municipal ante la declaratoria de emergencia (Secretaria de Educación Recreación y Deporte, s.f.)

Se implementa el Plan Educativo con el objetivo garantizar una educación de calidad y proveer herramientas pedagógicas, metodológicas, psicoemocionales y psicosociales, que fomenten y fortalezcan la construcción de un modelo educativo adaptable y contextualizado, que responda a las diversas necesidades del territorio nacional y permita garantizar el derecho a la educación en medio de la crisis (Ministerio de Educación del Ecuador MINEDUC, 2020),en conjunto con la radio y televisión, el Ministerio de Educación pone semanalmente a disposición del estudiante y padre de familia, diferentes fichas pedagógicas que evidencian contenidos prescindibles a las clases virtuales (clases a través del internet), modalidad que tuvo 
acogida por los estudiantes que tienen acceso a recursos tecnológicos, sin embargo, existen distintas percepciones y realidades.

Uno de los factores que juegan un papel importante dentro del contexto actual son los procesos de enseñanza-aprendizaje, como afirma Contreras (1994), el cual se entiende como el sistema de comunicación intencional que se produce en un marco institucional y en el que se generan estrategias encaminadas a provocar el aprendizaje, existiendo una relación de dependencia ontológica entre las tareas de enseñanza y las tareas de aprendizaje, mediada por el flujo de tareas que establece el contexto institucional y dentro del cual se descubre el modo de realización de las tareas de aprendizaje. Son estas últimas las que pueden dar lugar a los aprendizajes. La comprensión de las mediaciones entre estos dos conceptos, de la dependencia, pero a la vez desigualdad y corte entre ambos, justifica el uso del concepto enseñanza-aprendizaje.

En el contexto ecuatoriano la etapa de planificación de las clases ha iniciado, mediante procedimientos distintos a los acostumbrados, es así que se ha implementado el teletrabajo. El uso de la Tecnología de la Información y Comunicación (TIC) se ha convertido en un componente fundamental de la educación en todo el mundo y la Tecnología para el Aprendizaje y Conocimiento (TAC) prometen mantener alejados a niños, jóvenes y adultos de los riesgos de contagio, mientras avanzan en sus estudios (Villafuerte, Bello, Cevallos, \& Vidal, 2020),esto se realiza con la utilización de diferentes recursos tecnológicos como WhatsApp, correo electrónico, aplicaciones de Google, televisión, radio y la implementación de nuevas estrategias metodológicas, que de uno u otra forma garanticen una educación de calidad.

La educación para muchas personas que habitan en zonas marginales y rurales, ha sido desatendida por parte de las autoridades. Sin embargo, existe un sin número de factores que influyen de manera negativa como el limitado acceso a equipos tecnológicos y al uso de internet, lo que impide que miles de niñas, niños y adolescentes puedan continuar con sus estudios (Carreño, 2020). Al respecto, estudios y trabajos recientes muestran que esta abrupta transición de la educación presencial a la virtual, ha puesto de manifiesto las desigualdades e inequidades que la sociedad en general venía evidenciando desde hace años (Quiroz Reyes, 2020).

Por otra parte, los niños de primaria requieren del acompañamiento de los padres para poder trabajar en línea y apropiarse de las herramientas que les permitan desarrollar las actividades previstas para cada grado escolar. Sin embargo, es imposible aseverar que todos los padres de familia se encuentran en condiciones intelectuales y psíquico-afectivas para manejar los contenidos de los programas y las tecnologías puestas en marcha para los diferentes grados. Tampoco se puede dar por sentado su capacidad para acompañar a los hijos en los aprendizajes; es decir, convertirse en pacientes y tenaces asistentes de los maestros para alen- 
tar a los alumnos, explicarles lo que no comprenden, ayudarlos a organizar su tiempo, interpretar las orientaciones orales y escritas que les plantean, reconocer y valorar sus esfuerzos, entre otras cosas (Ducoing Watty, 2020).

Es probable que la educación temprana y el aprendizaje fundamental durante los primeros años de la escuela primaria se vean afectados, por otra parte, cuando reabran las escuelas, es probable que los niños de los sectores rurales se encuentren incluso más atrasados en comparación con sus pares (Halsey, Rogers; Shwetlena, Sabarwal, 2020).

Ahora bien, se pretende analizar el impacto de la emergencia sanitaria ocasionada por la pandemia COVID-19 en la educación primaria rural, determinando las brechas que existen en la educación virtual, los desafíos de la escuela Kiwanis en el contexto de la pandemia COVID- 19, enfocados principalmente en las tecnologías de la información y comunicación, y el análisis de la factibilidad de las fichas pedagógicas implementadas en el sistema educativo por el Ministerio de Educación.

\section{MATERIAL Y MÉTODO}

En concordancia con la metodología establecida se realiza la elección del enfoque mixto como menciona Guelmes Valdés \& Nieto Almeida (2015) este es un proceso que recolecta, analiza y vincula datos cuantitativos y cualitativos en una misma investigación para responder a un planteamiento, y justifica la utilización de este enfoque en su estudio considerando que ambos métodos se entremezclan en la mayoría de sus etapas (pág. 24).

Por lo que la investigación presenta un enfoque cualitativo porque el problema está en el contexto educativo inmerso en el proceso de enseñanza-aprendizaje de los educandos en una forma virtual, pero a la vez predomina un enfoque cuantitativo debido a que se basa en la recolección de datos numéricos, estandarizando y cuantificando, para el análisis e interpretación de los mismos, teniendo la posibilidad de generar los resultados obtenidos en la investigación de manera numérica.

La investigación se lleva a cabo en la Escuela Fiscal Kiwanis ubicada en el barrio Panzaleo, parroquia Machachi, cantón Mejía y provincia de Pichincha, la cual va dirigida a veinte personas (padres de familia), correspondiente al 100\% de la población en análisis, por lo que no requiere utilizar muestras ya que el número de encuestados es inferior al requerido.

La técnica que se estableció es la encuesta con su respectivo cuestionario, consta de cinco interrogantes, con lo que se pretende evidenciar la realidad de la educación primaria en los 
sectores rurales, lo que aportará a contemplar de manera real la situación que vive el sistema educativo fiscal primario, y se analizará si es factible o no la ejecución del Plan Educativo Covid-19 implementado por el Ministerio de Educación durante la emergencia sanitaria.

\section{RESULTADOS}

Según la encuesta realizada, se arrojan los siguientes resultados.

\section{INSTRUCCIÓN ACADÉMICA}

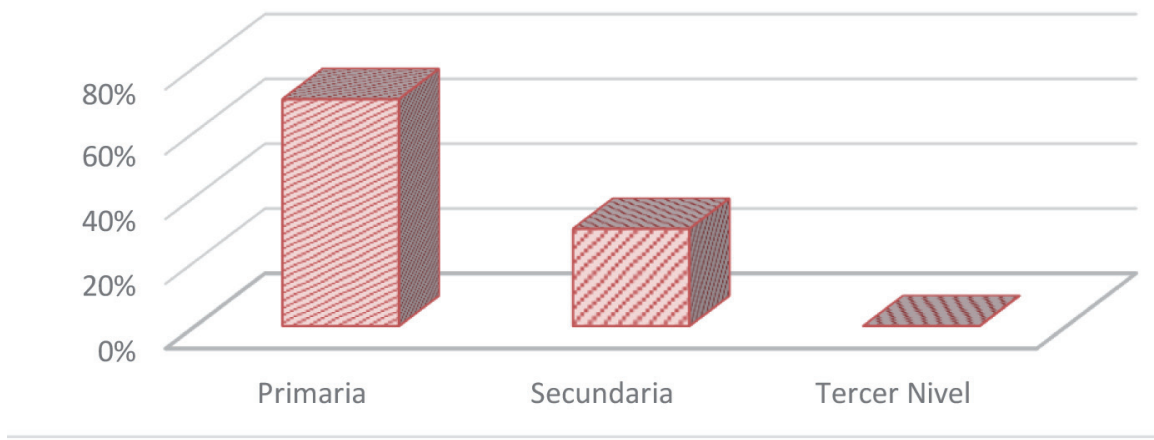

Figura 1. Nivel de Instrucción de los padres de familia. Fuente: elaboración propia

Como se evidencia en la Figura 1, en el sector rural la mayoría padres de familia tienen instrucción primaria lo cual limita su participación en los procesos de enseñanza-aprendizaje de sus hijos, complicándoles el uso de la tecnología. Muchos padres mencionaron que no sentían la necesidad de adquirir una computadora porque tenían una enseñanza presencial y no se previa que en algún momento iba a darse una pandemia.

\section{ACCESO A INTERNET}

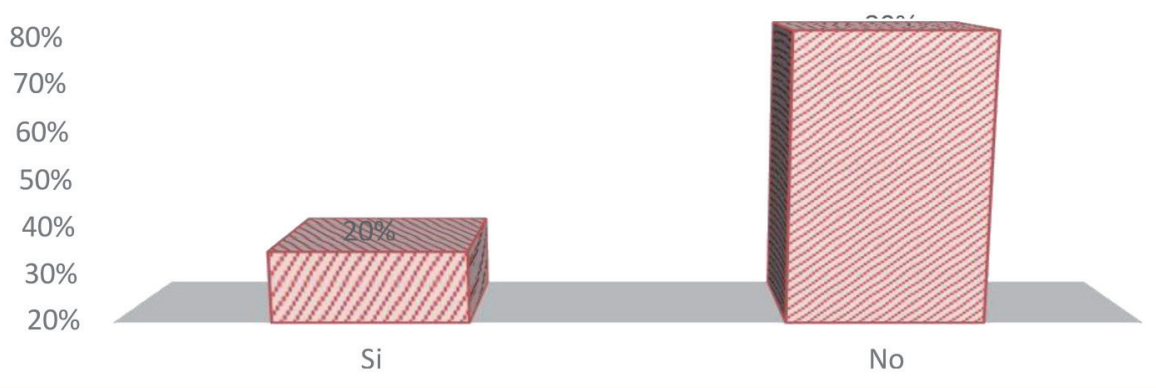

Figura 2. Disponibilidad de Internet. Fuente: elaboración propia 
En el sector rural que se aplicó esta técnica la mayoría son empleados de haciendas, viven de la agricultura y tienen falta de conocimiento para contratar servicios de internet, considerando que antes de la pandemia sus hijos gozaban de una educación presencial, por lo que no sentían la necesidad de adquirir este servicio básico.

\section{USO FICHAS PEDAGÓGICAS}

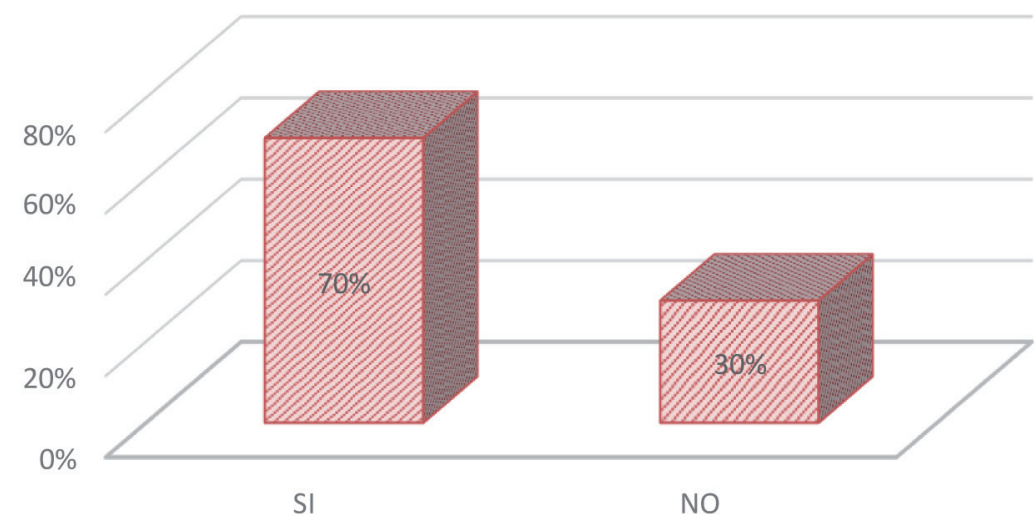

Figura 3. Utilidad de las fichas pedagógicas semanales. Fuente: elaboración propia

Los padres de familia en su mayoría utilizan las fichas pedagógicas como un recurso emitido por el Ministerio de Educación dentro del Plan Educativo Covid-19, para el proceso de enseñanza-aprendizaje de sus representados, enviadas por el docente a través de WhatsApp, cabe mencionar que en las fichas constan las respectivas indicaciones para su desarrollo, sin embargo los estudiantes no adquieren un aprendizaje significativo, debido a que no existe el acompañamiento de un facilitador y solo el $20 \%$ de estudiantes tienen acceso a internet.

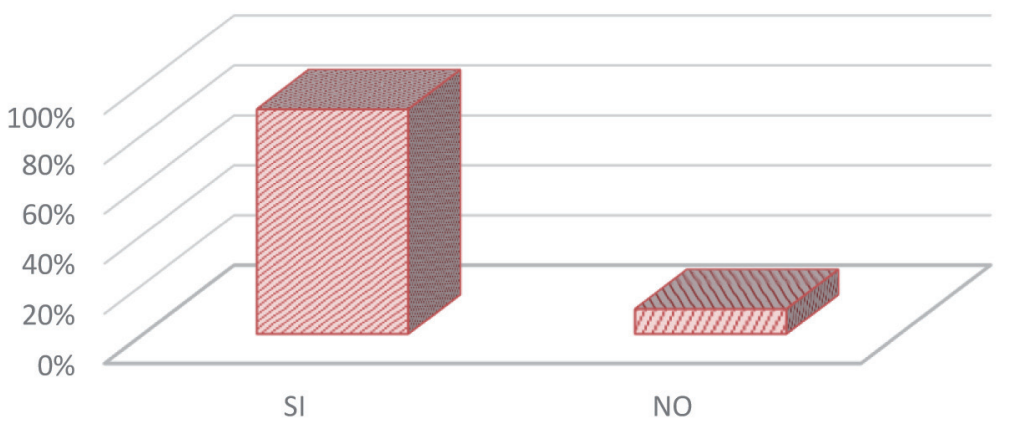

Figura 4. Incidencia en la pandemia en el ámbito educativo. Fuente: elaboración propia

Como se muestra en la Figura 4, un alto porcentaje de la población manifiesta que la pandemia impacto en la educación al darse la transición de modalidad presencial a virtual, ya que 
las familias no estaban preparadas para esta transformación mundial menos a un cambio en el sistema educativo, puesto que la gran mayoría de personas tienen un nivel bajo de educación.

\section{DISCUSIÓN}

Gran parte de esta problemática es la transición de educación presencial a educación virtual como menciona De Vicenci (2020) millones de docentes y estudiantes debieron reaprender una nueva manera de acceder al conocimiento y de interactuar en el ciberespacio. Incluso los más resistentes a la cultura digital tuvieron que involucrarse en este proceso de aprendizaje y apropiación tecnológica.

Además, la educación rural se ve afectada por falta de recursos tecnológicas como afirma Unicef (2020) En Ecuador, solo el 37\% de los hogares tiene acceso a internet, lo que significa que 6 de cada 10 niños no pueden continuar sus estudios a través de plataformas digitales. La situación es más grave para los niños de zonas rurales, solo el $16 \%$ de los hogares tiene este servicio. Asimismo, se determinó que en la escuela Kiwanis del Barrio Panzaleo, los padres de familia se enfrentan a varios retos, como la falta de servicio de internet, las fallas en la conectividad y la recesión económica en sus hogares, por la modalidad de educación virtual, sumada a la crisis económica y social que ha dejado la pandemia del COVID- 19 a nivel mundial.

Otro aspecto es que los representantes no cuentan con las habilidades digitales necesarias para dar continuidad a las actividades escolares y en los casos más extremos algunos de ellos tienen una formación escolar menor a la que cursan sus hijos, lo cual les imposibilita para apoyarles en las tareas escolares.

Los países se enfrentaron al cierre repentino de las instituciones como manifiesta Cóndor Herrera (2020) es frente a estas adversidades que el modelo educativo se ha visto en la obligación de desarrollar el Plan Educativo Covid-19 con varios recursos como las fichas pedagógicas que son herramientas para que cada estudiante continúe con el desarrollo de su proceso educativo mismas que son utilizadas por la gran mayoría de los niños en la escuela Kiwannis, ya que un gran porcentaje tiene obstáculos para acceder al modelo de educación virtual, este recurso permite ejecutar actividades que aborden las diferentes áreas del conocimiento el proceso pedagógico (Cruz Vasco, 2020) lo cual es un desafío en las condiciones de igualdad social. Escuelas privadas y públicas actualmente no cuentan con la infraestructura tecnológica ni la profundización digital suficiente para poder enfrentar una educación virtual. 
La pandemia del COVID-19 ha traído cambios drásticos en el sistema educativo tras la suspensión de clases desde marzo y el cambio de modalidad en la educación, lo que ha ocasionado grandes desafíos para familias enteras, sin embargo, esta transformación requiere un proceso de tratamiento desde sus cimientos y por ende se hace necesario que la escuela Kiwanis realice un cambio desde sus bases. Para Hurtado Talavera (2020) no basta con implementar recursos tecnológicos, se hace necesario que la escuela se reinvente y realice las reformas pertinentes para una verdadera cultura digital que respondan a las exigencias y requerimientos de los educandos en sus distintos contextos y realidades para de esta manera garantizar una educación de calidad.

\section{CONCLUSIONES}

Esta investigación ha permitido analizar y al mismo tiempo, determinar los puntos de vista de los padres de familia en la educación de sus hijos tras la pandemia del COVID-19 en donde es claro evidenciar la falta de recursos que existe en el sector rural y la limitación al acceso de internet lo cual impide continuar con el proceso educativo.

Además, se observó que los padres cambiaron un rol importante en la formación académica de sus hijos, ya que se han visto en la necesidad de orientar el proceso educativo, pero es lamentable conocer que la gran mayoría solo han terminado la primaria por lo que se les dificulta ser guías en el aprendizaje.

También, es muy desalentador determinar que los pilares del hogar no estaban preparados para una educación virtual, por la evolución de las nuevas tecnologías que en la actualidad el mundo está cursando, ya que en Sudamérica la mayoría de países tiene limitaciones para este tipo de enseñanza.

Finalmente, al implementar las fichas pedagógicas, no se consideró los problemas educativos que los alumnos ya acarreaban en años anteriores, las circunstancias sociales y económicas de los sectores rurales y ahora sumado a los desafíos que enfrenta el cambio de modalidad de una educación presencial a una educación virtual, se puede deducir que no es posible un proceso efectivo de enseñanza-aprendizaje, es decir, que la transmisión de contenidos en entornos virtuales no puede darse con las suficientes garantías.

La educación sufrió desafíos por la implementación de nuevas tecnologías, dando oportunidad a los docentes para innovar sus aulas con las herramientas que actualmente se han desarrollado, lo cual podría ser una oportunidad de aprender sobre las nuevas herramientas tec- 
nológicas para la educación y los procesos de emergencia, además, optimizar los procesos de enseñanza- aprendizaje con una utilización mixta de los métodos tradicionales y los digitales.

Se recomienda que las autoridades de la Escuela Kiwanis elaboren un proyecto de capacitación para los padres de familia sobre tres puntos principales: charlas motivacionales sobre retos y oportunidades durante la pandemia, conceptos básicos de uso de herramientas virtuales y acompañamiento de los procesos de enseñanza-aprendizaje a los educandos, el mismo que deberá ser implementado con la aprobación y apoyo del Distrito Educativo.

\section{REFERENCIAS BIBLIOGRÁFICAS}

Carreño, M. (06 de 10 de 2020). Tras la pandemia, en Ecuador bajó la calidad de la educación.

Obtenido de Pastoral Social Caritas del Ecuador: https://www.caritasecuador.org/2020/10/pandemiacalidad-educacion/

Cóndor Herrera, O. (04 de 05 de 2020). Educar en tiempos de Covid. CienciAmérica, 9(2).

Contreras Domingo, J. (1994). LA DIDÁCTICA Y LOS PROCESOS DE ENSENANZA-APRENDIZAJ E. Apple, 13-38. Obtenido de http://rubenama.com/historia unam/lecturas/contreras ensenanza cap1.pdf

Cruz Vasco, A. (2020). Paln educativo aprendamos juntos en casa. Quito: Ministerio de Educación. Obtenido de https://educacion.gob.ec/wp-content/uploads/downloads/2020/08/Plan-EducativoAprendamos-Juntos-en-Casa.pdf

De Vicenci, A. (2020). Del aula presencial al aula virtual universitaria en contexto de pandemia de COVID-19. Avances de una experiencia universitaria en carreras presenciales adaptadas a la modalidad virtual. Revista académica electrónica semestral, 8(16), 67-71. http://200.32.31.164:9999/ ojs/index.php/debate- universitario/article/view/238/242

Ducoing Watty, P. (2020). Una expresión de la desigualdad en educación. En J. Aguilar, A. Alcántara, F. Álvarez, R. Amador, C. Barrón, M. T. Bravo, R. Castañeda, Educación y pandemia Una visión académica (págs. 58-59). México: iiSUE. Obtenido de https://api.includere.co/ uploads/1591109044_UNAM\%20educacion_pandemia.pdf\#page $=30$

El País. (16 de 06 de 2020). Ecuador: la educación online desde casa es imposible e injusta. LA CRISIS DE LA COVID-19. Obtenido de https://elpais.com/elpais/2020/06/12/planeta futuro/1591955314_376413.html

Guelmes Valdés, E. L., \& Nieto Almeida, L. E. (2015). Algunas reflexiones sobre el enfoque mixto de la investigación pedagógica en el contexto cubano. Revista Científica de la Universidad de Cienfuegos Universidad y Sociedad, 24. Obtenido de http://scielo.sld.cu/pdf/rus/v7n1/rus03115.pdf 
Halsey, Rogers; Shwetlena, Sabarwal. (2020). COVID-19: IMPACTO EN LA EDUCACIÓN Y RESPUESTAS DE POLÍTICA PÚBLICA. Grupo Banco Mundial. Obtenido de http://pubdocs.worldbank.org/ en/143771590756983343/Covid-19-Education-Summary- esp.pdf

Hurtado Talavera, F. J. (2020). LA EDUCACIÓN EN TIEMPOS DE PANDEMIA: REVISTA ARBITRADA DEL CENTRO DE INVESTIGACIÓN Y ESTUDIOS GERENCIALES, 176-

187. Obtenido de http://www.grupocieg.org/archivos revista/Ed.44(176- 187)\%20Hurtado\%20Tavale$\underline{\text { ra articulo id650.pdf }}$

Ministerio de Educación del Ecuador MINEDUC. (2020). Plan Educativo COVID-19. Obtenido de https:// educacion.gob.ec/plan-educativo-covid-19/

Ministerio de Educación MINEDUC. (12 de 03 de 2020). Acuerdo Ministerial No MINEDUC- MINEDUC2020-00013- $A$. Obtenido de https://educacion.gob.ec/plan-educativo-covid- 19-se-presento-el16-de-marzo/

Ministerio de Educación MINEDUC. (2020). Comunicado Oficial. Obtenido de https://educacion.gob. ec/comunicado-oficial-suspension-de-las-actividades-academicas-para-los-estudiantes-paraprecautelar-la-salud-de-la-comunidad-educativa/

Organización Mundial de la Salud OMS. (2019). Brote de enfermedad por coronavirus (COVID-19). https://www.who.int/es/emergencies/diseases/novel-coronavirus-2019

Organización Panamericana de Salud OPS. (2020). Enfermedad del Coronavirus (COVID-19). https:// www.paho.org/es/tag/enfermedad-por-coronavirus-covid-19

Quiroz Reyes, C. (2020). «Pandemia Covid-19 e Inequidad Territorial: El Agravamiento de las Desigualdades Educativas en Chile». Revista Internacional de Educación para la fusticia Social, 9(3).

Secretaria de Educación Recreación y Deporte. (s.f.). Lineamientos para la implementación del Plan Educativo Municipal ante la declaratoria de enjmergencia COVID-19. Obtenido de http://www. educacion.quito.gob.ec/colegios/nuevedeoctubre/index.php/micolegio/inclusi on-educativa/2plan-de-emergencia/41-plan-de-contingencia-covid-19

Unicef Ecuador. (04 de 05 de 2020). Unicef para cada niño. Obtenido de https://www.unicef.org/ecuador/historias/covid-19-c\%C3\%B3mo-asegurar-el-aprendizaje-de-los-ni\%C3\%B1os-sin-accesointernet\#: :text=En\%20Ecuador\%2C\%20solo\%20el\%2037,los\%20hogares\%20tiene $\% 20$ este $\% 20$ $\underline{\text { servicio. }}$

Villafuerte, H., Bello, P., Cevallos, P., \& Vidal, B. (2020). Rol de los docentes ante la crisis del Covid 19, Una mirada desde el enfoque Humano. REFCaIE, 134-150. 